\title{
LIGO/Virgo Black Holes from a First Order Quark Confinement Phase Transition
}

\author{
Hooman Davoudiasl" \\ Physics Department, Brookhaven National Laboratory, Upton, New York 11973, USA
}

(Received 11 March 2019; revised manuscript received 9 August 2019; published 6 September 2019)

\begin{abstract}
We propose that $\mathcal{O}\left(10 M_{\odot}\right)$ black holes observed by LIGO/Virgo originate from a first order phase transition at a temperature $T_{*} \lesssim 100 \mathrm{MeV}$, associated with $\mathrm{QCD}^{\prime}$ - an early Universe deformation of the standard model QCD. This is realized by keeping the quark masses small compared to confinement scale down to $T \sim T_{*}$, making $\mathrm{QCD}^{\prime}$ transition first order. We implement this scenario using a light scalar that could potentially be a good dark matter candidate.
\end{abstract}

DOI: 10.1103/PhysRevLett.123.101102

Direct observations of gravitational waves from mergers of compact stellar objects by the LIGO/Virgo collaborations mark the dawn of a new era in astronomy [1]. An interesting feature of the extant binary merger data is that they apparently point to a population of $\mathcal{O}\left(10 M_{\odot}\right)$ black holes [2], with $M_{\odot} \approx 2 \times 10^{33} \mathrm{~g}$ the Solar mass, which could have an astrophysical origin [3,4]. However, apart from the potential astronomical information gleaned from these impressive measurements, one is compelled to consider if this new probe can shed light on fundamental questions in particle physics and cosmology. One of the early attempts soon after the first detection by LIGO was to consider whether the observed merger was of $\sim 30 M_{\odot}$ primordial black holes (PBHs) that constitute dark matter [5]. Such PBHs are constrained by various observational data [6] and may make up only a fraction $\lesssim \mathcal{O}(10 \%)$ of the cosmic dark matter budget [7]. Yet, it is still worthwhile to inquire whether such a population of objects can be of primordial origin, though they may not be the main component of dark matter.

It has long been argued that the probability of forming a population of $\sim M_{\odot} \mathrm{PBHs}$ is considerably enhanced during the QCD confining phase transition [8,9]. The PBH mass scale is set by the size of the horizon at the time of the transition which roughly corresponds to a temperature of $T_{\mathrm{QCD}} \approx 160 \mathrm{MeV}$ [10]. Nonetheless, the typical PBH mass in this case is a factor of $\mathcal{O}(10)$ smaller than that suggested by the LIGO/Virgo data. Also, in the standard model (SM) the QCD phase transition is not first order [10], and hence not as efficient for the purposes of generating PBHs. The underlying reason is that during a first order phase transition the speed of sound tends to zero, and hence

Published by the American Physical Society under the terms of the Creative Commons Attribution 4.0 International license. Further distribution of this work must maintain attribution to the author(s) and the published article's title, journal citation, and DOI. Funded by SCOAP ${ }^{3}$. the pressure response of the fluid vanishes and does not counterbalance the collapse of horizon-sized primordial overdensities [11]. While the pressure response is expected to be lower during the SM QCD transition, the effect would not provide the same efficiency as a first order transition. Also, the standard confinement would dominantly yield $\mathcal{O}\left(M_{\odot}\right)$ PBHs [12].

Theoretical analyses which provide an effective description of QCD at low energies, based on its underlying symmetries, suggest that the confining transition would be first order if the number of light quarks $N_{f} \geq 3$ [13], at the onset of confinement. The three lightest quarks in the SM are the up, the down, and the strange quarks. While the masses of the up and the down quarks are small compared to the transition temperature $T_{\mathrm{QCD}}$, the strange quark mass $m_{s} \approx 100 \mathrm{MeV}$ is not far from $T_{\mathrm{QCD}}$, and therefore the strange quark cannot be considered nearly massless at such temperatures. Hence, the condition for a first order phase transition is not satisfied in the SM, in agreement with results from computer simulations of $\mathrm{QCD}$, using lattice gauge theory techniques [10]. We note that a lattice QCD confirmation of the prediction in Ref. [13] is still under investigation [14].

In this work, we entertain the possibility that the $\mathcal{O}\left(10 M_{\odot}\right)$ population of black holes points to a first order confining phase transition of $\mathrm{QCD}^{\prime}-\mathrm{a}$ modification of the SM QCD in the early Universe with the number of light quarks $N_{f}=6$ at the onset of the transition. Given that additional light quarks drive the scale of QCD' confinement to lower values, one then expects $T_{\mathrm{QCD}} \lesssim 160 \mathrm{MeV}$ in this scenario, corresponding to a lager Hubble volume and hence larger typical PBH masses. Obviously, our mechanism could also be used to have fewer light quarks, $3 \leq N_{f} \leq 6$, at the onset of confinement which would still be expected to lead to a first order transition. However, we will focus on the maximal case $N_{f}=6$, as it could yield the lowest $T_{\mathrm{QCD}}$ and hence the heaviest PBHs.

If one arranges for the Higgs field to get a vacuum expectation value (VEV) after the confining transition, at 
$T \lesssim 100 \mathrm{MeV}$, one could have $N_{f}=6$ light quarks during the transition. Here, quark condensation $\langle\bar{q} q\rangle \neq 0$ breaks electroweak symmetry. This model could result in the desired phase transition, but it is expected to entail a period of inflation, due to the relatively large Higgs vacuum energy. (We thank V. Vaskonen for emphasizing this effect, and its associated problems, in such a scenario.) In the inflationary phase, the Hubble constant is typically governed by weak scale energies, and thus the formation of $\sim 10 M_{\odot}$ does not appear feasible in such a scenario. While not directly relevant to the subject of this work, this scenario could potentially yield interesting cosmology; we will leave the study of that possibility for future work.

To achieve a first order confining phase transition, leading to $\sim 10 M_{\odot} \mathrm{PBHs}$, we consider a model in which the dynamics of a light scalar suppresses quark masses before the $\mathrm{QCD}^{\prime}$ transition, but results in the measured values afterwards. This scenario does not entail the above inflationary period, as Higgs condensation takes place at the conventional temperature of $T \sim 100 \mathrm{GeV}$. (The value of the QCD vacuum energy is not precisely known; its estimates cover a fairly broad range $[15,16]$. That range could allow for a somewhat lower $T_{\mathrm{QCD}}$, corresponding to a first order confining transition with $N_{f}=6$, without leading to inflation.) Generally speaking, we will not address various potential tunings that are required to realize the parameters of the models we will discuss. Some of these questions may be addressed in ultraviolet completions of the effective theories we consider, but that question is beyond the scope of this work.

For some alternative ideas on achieving a first order confinement phase transition in the early Universe, see e.g., Refs. [17,18]. Reference [19] considers a first order confining phase transition at temperatures above the electroweak scale $\sim 100 \mathrm{GeV}$, due to a larger initial value of the coupling constant. The possibility of cold baryogenesis from strong $C P$ violation, with delayed electroweak symmetry breaking, was considered in Ref. [20]. In Ref. [21], the possibility of a first order confining transition with electroweak symmetry breaking after confinement has been considered and analyzed in some detail; see also Ref. [22]. (Reference [22] maintains that $M_{\odot} \mathrm{PBHs}$ form, as it implicitly assumes that no inflation takes place according to its adopted underlying theory. Instead, we implement a first order $\mathrm{QCD}^{\prime}$ transition through initially suppressed quark Yukawa couplings, assuming electroweak symmetry is broken, thereby avoiding possible difficulties related to weak scale vacuum energy domination.)

We also note that a first order confining phase transition may lead to inhomogeneous big bang nucleosynthesis (BBN) [23] if the distance $d_{n}$ between nucleated bubbles is larger than the proton diffusion length of order a few hundred cm [24-26]. However, estimates suggest that $d_{n} \lesssim 2 \mathrm{~cm}$, in a standard QCD transition [27]. Given this large margin of safety — by about 2 orders of magnitude - in the standard case, we may expect that in our nonstandard scenario with $N_{f}=6$, the value of $d_{n}$ would still be sufficiently small to avoid problems with BBN. To reach a firm conclusion on this question may require a dedicated nonperturbative analysis, which is beyond the scope of this work.

We will first discuss the effect of introducing additional light quarks at confinement transition, from a modelindependent point of view. The mass of PBHs, corresponding to the energy contained within the horizon during the radiation dominated era, can be approximated as [12]

$$
M_{\mathrm{PBH}}(T) \approx 2.4 \gamma M_{\odot} \sqrt{\frac{61.75}{g_{*}}}\left(\frac{160 \mathrm{MeV}}{T}\right)^{2},
$$

where $\gamma$ is an $\mathcal{O}(1)$ constant that, depending on the amplitude of primordial overdensities, can have values $\sim$ few $\times(0.1-1)[28,29]$ and $g_{*}$ is the relativistic degrees of freedom in the primordial plasma. Thus, Eq. (1) suggests that if $T_{\mathrm{QCD}}$ were well below $\sim 160 \mathrm{MeV}$ then one could take $M_{\mathrm{PBH}} \gg M_{\odot}$ to be the typical mass of PBHs formed during the $\mathrm{QCD}^{\prime}$ transition.

Next, we address the conditions for achieving a lower confinement temperature. We will examine how low $T_{\mathrm{QCD}}$ can be if in addition to the SM up and down quarks there are other quarks below $T_{\mathrm{QCD}}$ in the early Universe plasma. We will focus on the case when all SM quarks are light at $T_{\mathrm{QCD}}$, which is the case realized in a model we will propose below.

For a rough estimate, we first find the value of the standard QCD coupling constant $\alpha_{s}\left(\mu_{3}\right)$ at $\mu_{3} \sim 160 \mathrm{MeV}$, corresponding to confinement for the standard $N_{f}=3$ case. Then, we will find the scale $\mu_{6}$, corresponding to $N_{f}=6$ light quarks, by demanding $\alpha_{s}\left(\mu_{6}\right) \approx \alpha_{s}\left(\mu_{3}\right)$; we will use a one-loop approximation for the running. Obviously, this is not meant to be a precision treatment, but only an order of magnitude estimate for the value of $\alpha_{s}$ that would yield confinement.

At the one-loop order, we have

$$
\alpha_{s}^{-1}(\mu)=\alpha_{s}^{-1}\left(\mu_{0}\right)+\frac{2 N_{f}-33}{6 \pi} \ln \left(\mu_{0} / \mu\right),
$$

where $\mu_{0}$ is a reference scale. Let us take $\mu_{0}=m_{Z}$, where $m_{Z} \approx 91.2 \mathrm{GeV}$ is the mass of the $Z$ boson. We have $\alpha_{s}\left(m_{Z}\right) \approx 0.118(11)$ [30]. It follows from Eq. (2) that

$$
\delta \alpha_{s}=\frac{\delta N_{f}}{3 \pi} \alpha_{s}^{2} \ln \left(\mu_{0} / \mu\right) .
$$

Since in our setup the top quark will be light down to very small temperatures, we estimate that the effect of the extra quark, $\delta N_{f}=1$, on the value of $\alpha_{s}\left(m_{Z}\right)$, corresponding to running between the top mass and $m_{Z}$. Using the above expression, we then find $\left.\alpha_{s}\left(m_{Z}\right)\right|_{N_{f}=6} \approx 0.117$, which 
yields $\mu_{6} \sim 50 \mathrm{MeV}$ [by requiring $\alpha_{s}\left(\mu_{6}\right) \approx \alpha_{s}\left(\mu_{3}\right)$ ]. Hence, we may expect, with $N_{f}=6$, to have a confining phase transition scale $\sim 50 \mathrm{MeV}$. (Reference [31] finds that this temperature is $\sim 85 \mathrm{MeV}$. That result supports the idea that PBHs formed in our scenario are expected to be more massive than in the standard treatment, though the enhancement factor could be somewhat smaller than our estimate.) At the scales $\mu_{3}$ and $\mu_{6}$, in accord with the preceding discussion and using Eq. (2), we find $\alpha_{s}\left(\mu_{3}\right) \approx \alpha_{s}\left(\mu_{6}\right) \approx 5$ which corresponds to the onset of confinement.

Given that $\left(\mu_{3} / \mu_{6}\right)^{2} \sim 10$, at the order of magnitude level, we find

$$
\frac{\left.\sqrt{g_{*}} T_{\mathrm{QCD}}^{2}\right|_{N_{f}=3}}{\left.\sqrt{g_{*}} T_{\mathrm{QCD}}^{2}\right|_{N_{f}=6}} \sim \frac{\left.\sqrt{g_{*}}\right|_{N_{f}=3}}{\left.\sqrt{g_{*}}\right|_{N_{f}=6}} \times 10,
$$

where $\left.g_{*}\right|_{N_{f}=3}=61.75$ for the conventional case, and $\left.g_{*}\right|_{N_{f}=6}=93.25$ if all SM quarks are light at the transition, as could be the case in the model presented later on in this work. Using Eq. (1), we find

$$
M_{\mathrm{PBH}}\left(T_{\mathrm{QCD}} ; N_{f}=6\right) \sim 20 \gamma M_{\odot} .
$$

The above implies that a "cooler" confinement phase transition temperature could be the origin of the $\mathcal{O}\left(10 M_{\odot}\right)$ black holes observed by LIGO/Virgo.

To summarize the preceding discussion, we have argued that in the presence of $N_{f}=6$ light quarks during confinement, (i) a significant enhancement in the efficiency of PBH production can be achieved due to a first order phase transition, and (ii) a boost of the PBH masses by about an order of magnitude to $\mathcal{O}\left(10 M_{\odot}\right)$ due to a lower transition temperature can result.

Here, we do not address the relic abundance of the PBHs. If PBHs comprise a fraction $f$ of the cosmic dark matter budget, some estimates suggest that $f \sim 0.001$ [32-35] is needed in order to be consistent with the LIGO/Virgo merger signal. The value of $f$ depends on the probability distribution for the energy density contrast parameter $\delta$ (see e.g., Ref. [12]). Our scenario is only concerned with the efficiency of producing $\mathcal{O}\left(10 M_{\odot}\right)$ PBHs; we have implicitly assumed that the required distribution of $\delta$ was realized in the early Universe. We will next consider models that could in principle implement the above scenario, where $N_{f}=6$ quarks are light at the confining $\mathrm{QCD}^{\prime}$ transition, making it first order.

We aim to present a scenario that would avoid subtleties associated with an inflationary period from weak scale vacuum energies. Here, we assume that electroweak symmetry is broken in the usual fashion, at $T \sim 100 \mathrm{GeV}$, but quarks remain lighter than $\sim 100 \mathrm{MeV}$. To see how this could happen, let right-handed SM quarks $q_{R}$ and a light scalar field $\varphi$ be odd under a $\mathbb{Z}_{2}$. Then, we need terms of the form

$$
\frac{\varphi H \bar{Q}_{L} q_{R}}{\Lambda_{\varphi}}
$$

where $Q_{L}$ and $H$ are the SM quark and Higgs doublets, respectively. We assume that $\varphi$ starts at $\varphi=0$. This can be the result of a period of high temperature epoch followed by inflation that locks $\varphi$ at the origin. Phenomenologically, we expect that $\Lambda_{\varphi} \gtrsim 1 \mathrm{TeV}$, given that the SM seems to be a good effective theory up to this energy scale.

In order for the $\varphi$ to produce the correct top mass, we need the final value of $\varphi \sim \Lambda_{\varphi}$. A scalar field starts to track its potential once the Hubble scale is about the size of the scalar mass. Hence, for $\varphi$ to evolve to its final value only after $\mathrm{QCD}^{\prime}$ confinement, we would generically need $m_{\varphi} \lesssim 10^{-12} \mathrm{eV}$, corresponding to the Hubble scale $\mathcal{H} \sim T^{2} / M_{P}$ at that era, where the Planck mass $M_{P} \approx 1.2 \times 10^{19} \mathrm{GeV}$. For such a light field to avoid causing severe deviations from Newtonian gravity, its couplings to nucleons must be very tiny. However, the largest plausible value of the suppression scale in Eq. (6) is $\Lambda_{\varphi} \sim M_{P}$. One would then end up with a light field that starts with an initial amplitude of oscillations of $\mathcal{O}\left(M_{P}\right)$ and a mass of $\sim 10^{-12} \mathrm{eV}$ resulting in an energy density $\rho \sim$ $\left(m_{\varphi} \Lambda_{\varphi}\right)^{2} \sim 10^{32} \mathrm{eV}^{4}$ at $T \sim T_{\mathrm{QCD}}$, which would be too large for viable dark matter. Also, the strength of the low energy coupling of $\varphi$ to nucleons will end up being $\mathcal{O}\left(10^{-20}\right)$ or so [36], which is too large by a factor of $\sim 10^{4}$ [37]. To address this issue, one could imagine adding various particles at high scales that could lead to cancellations among the effective $\varphi$-gluon couplings. In what follows, we will sketch such a model.

Let us assume that there is a scalar $\Phi$ of mass $m_{\Phi} \gtrsim$ $100 \mathrm{GeV}$ and $\operatorname{VEV}\langle\Phi\rangle \sim 10 m_{\Phi}$. We also consider a pair of vectorlike SM color triplet fermions $F_{i}, i=1,2$, with hypercharge quantum numbers $Q_{Y}$ of $q_{R}$, and a light scalar $\phi$ with a large initial value. It is assumed that $\mathbb{Z}_{2}(\Phi)=\mathbb{Z}_{2}\left(q_{R}\right)=-\mathbb{Z}_{2}\left(F_{i}\right)=-1$. Note that $F_{i}$ have either $Q_{Y}=+2 / 3$ or $-1 / 3$. We would then need both types to achieve mass suppression for all six SM quarks. With only these assumptions, couplings of $\Phi$ to leptons are forbidden by the $\mathbb{Z}_{2}$ symmetry. We can then write down the following interactions [correct $S U(2)_{L}$ structure implicit]:

$y_{q} \Phi \bar{F}_{i L} q_{R}+\xi_{q} H \bar{Q}_{L} F_{i R}+M_{F} \bar{F}_{i} F_{i}+\lambda_{i} \phi \bar{F}_{i} F_{i}+$ H.c.,

where we have suppressed the flavor index for quarks. Given the above setup, the following effective operator can be obtained

$$
y_{q} \xi_{q} \sum_{i} \frac{\Phi}{\Lambda_{\Phi i}} H \bar{Q}_{L} q_{R}
$$

The scale $\Lambda_{\Phi i}=M_{F}+\lambda_{i} \phi$, where $M_{F}$ is the $F_{1,2}$ vectorlike mass for $\phi \rightarrow 0$, at late times. The effective $\phi$-dependent 
Yukawa coupling of the SM quarks to the Higgs field, $\lambda_{q} H \bar{Q}_{L} q_{R}$, is then given by

$$
\lambda_{q}(\phi)=\sum_{i} \frac{y_{q} \xi_{q}\langle\Phi\rangle}{M_{F}+\lambda_{i} \phi}
$$

where $y_{t} \xi_{t}\langle\Phi\rangle \approx M_{F} / 2$, for we need to recover $\lambda_{t}(0) \approx 1$ at late times. We then see, from Eq. (9), that $\lambda_{q} \ll 1$ for $\left|\lambda_{i}\right| \phi \gg M_{F}$.

The potential for $\phi$ is simply given by its mass term $(1 / 2) m_{\phi}^{2} \phi^{2}$. Assuming that $m_{\phi} \sim 10^{-12} \mathrm{eV}$, the field $\phi$ will start tracking its potential to $\phi=0$ after the phase transition at $T_{\mathrm{QCD} /} \lesssim 100 \mathrm{MeV}$. If the initial $\phi$ energy density $\rho\left(T_{\mathrm{QCD} \prime}\right) \sim m_{\phi}^{2} \phi^{2} \sim 10^{24} \mathrm{eV}^{4}$, by the time of matter-radiation equality at $T_{m r} \sim 1 \mathrm{eV}$ in standard cosmology, $\rho$ will be diluted by $\left(T_{m r} / T_{\mathrm{QCD}}\right)^{3} \sim 10^{-24}$ to achieve the standard value $\rho\left(T_{m r}\right) \sim \mathrm{eV}^{4}$. Hence, $\phi$ could be a viable dark matter candidate for $\phi_{i} \sim 10^{15} \mathrm{GeV}$, where $\phi_{i}$ is the initial value of $\phi$.

As before, the above setup would lead to severe deviations from Newtonian gravity if $\phi$-nucleon coupling $y_{n} \gtrsim$ $10^{-24}$ [37]. This could, for example, be mediated by top quark mixing with $F_{1,2}$ from the interactions [Eq. (7)]. We want $M_{F}$ to be small compared to $\left|\lambda_{i}\right| \phi$ and $y_{t} \xi_{t}\langle\Phi\rangle \approx M_{F} / 2$. On the other hand, $\phi \lesssim 10^{15} \mathrm{GeV}$ from the above discussion, so that $\phi$ oscillations do not overclose the Universe. Hence, we conclude that the typical $\phi$ nucleon coupling would be too large, unless there is a cancellation among various contributions. We find that if the couplings $y_{q}$ and $\xi_{q}$ are equal, while $\lambda_{1}+\lambda_{2}=0$, then the contributions of loop diagrams mediated by $F_{1}$ and $F_{2}$ that induce $\phi$-nucleon coupling yield $y_{n}=0$ today, corresponding to $\phi=0$ (see, e.g., Ref. [38] for a possible implementation of such interactions in a string theory context). One could have a sufficiently small $y_{n}$ if the model parameters have only tiny deviations from the above assumed values. We also note that with the above assumptions, the couplings of $\phi$ to charged leptons, through 2-loop diagrams involving $F_{i}$ and photon, can be set to zero or sufficiently small.

Let $\lambda_{1}=-\lambda_{2}=\lambda$. We then find that $\lambda_{q}\left(\phi_{i}\right) \sim$ $-y_{q} \xi_{q}\langle\Phi\rangle M_{F} /\left(\lambda \phi_{i}\right)^{2}$. To have light top quarks during the transition, we require that $\lambda_{t}\left(\phi_{i}\right) \lesssim 10^{-3}$. If the $\phi$ condensate is a viable dark matter candidate, then we obtain $M_{F} / \lambda \lesssim 5 \times 10^{13} \mathrm{GeV}$.

We then conclude that the above model provides a reasonable picture for how the quark-gluon confining phase transition could be first order, at a somewhat lower temperature, while providing a possible dark matter candidate from $\phi$ oscillations. We note that the required mass for $\phi$ can be naturally obtained after the first order transition if there is a Planck scale coupling $\sim \phi^{2} \operatorname{Tr}\left[G_{\mu \nu} G^{\mu \nu}\right] / M_{P}^{2}$, where $G_{\mu \nu}$ is the gluon field strength tensor. The value of the gluon condensate is estimated to be $\left\langle\alpha_{s} G G\right\rangle \sim 10^{-2} \mathrm{GeV}^{4}$ [39-41], with $\alpha_{s}$ the strong coupling constant. In our setup quarks confine at a lower scale and we estimate $\left\langle\alpha_{s} G G\right\rangle \sim$ $10^{-3} \mathrm{GeV}^{4}$ for $N_{f}=6$ light quarks, which yields the right order of magnitude for $m_{\phi}$.

Here we would like to describe some of the potential phenomenological predictions of the above scenarios. Assuming that the above setup can realize a first order confinement transition, motivated by the masses of the black holes observed by LIGO/Virgo, we can expect primordial gravitational waves corresponding to an epoch $T_{\mathrm{QCD} /} \sim 50-100 \mathrm{MeV}$. Such signals may be detectable using pulsar timing arrays. This idea was first discussed in Ref. [42] and more recently studied in Ref. [43]; see also Ref. [44].

One could possibly consider values of $M_{F} \gtrsim \mathrm{TeV}$. In that case, the degenerate fermions $F_{i}$ may be within the reach of the LHC. From the interactions [Eq. (7)] one could expect their main decay channels to be $F_{i} \rightarrow H q, F_{i} \rightarrow \Phi q$, $F_{i} \rightarrow Z q$, and $F_{i} \rightarrow W q$, where $q=t, b$ depending on the hypercharge of $F_{i}$. If $m_{\Phi}>2 m_{t}$, a possible decay channel for $\Phi$ would be into a pair of top quarks.

To summarize, motivated by the $\mathcal{O}\left(10 M_{\odot}\right)$ black holes observed by LIGO/Virgo in binary mergers, we entertained the possibility that the quark-gluon confinement phase transition was first order due to the effect of 6 light quarks. The larger number of light quarks, compared to the standard case, pushes the transition temperature below $\sim 100 \mathrm{MeV}$. The first order nature of the transition significantly improves the likelihood of forming primordial black holes and its lower temperature suggests that these black holes can potentially be as heavy as $\sim 10 M_{\odot}$, compared to $\sim M_{\odot}$ for the standard QCD transition. We presented a model that could potentially realize the above scenario, lead to standard QCD after the transition, and yield a good dark matter candidate, which is a light scalar of mass $\sim 10^{-12} \mathrm{eV}$.

We thank S. Dawson, P. Petreczky, R. Pisarski, and P. Serpico, for discussions and comments. The author is grateful to D. Morrissey for helpful comments on an earlier version of the manuscript and V. Vaskonen for pointing out problems related to inflation therein. This work is supported by the United States Department of Energy under Grant Contract No. DE-SC0012704.

*hooman@bnl.gov

[1] B. P. Abbott et al. (LIGO Scientific and Virgo Collaborations), Phys. Rev. Lett. 116, 061102 (2016).

[2] (LIGO Scientific and Virgo Collaborations), arXiv:1811 12907.

[3] K. Belczynski, T. Bulik, C. L. Fryer, A. Ruiter, J. S. Vink, and J. R. Hurley, Astrophys. J. 714, 1217 (2010).

[4] M. Spera, M. Mapelli, and A. Bressan, Mon. Not. R. Astron. Soc. 451, 4086 (2015). 
[5] S. Bird, I. Cholis, J. B. Muñoz, Y. Ali-Haïmoud, M. Kamionkowski, E. D. Kovetz, A. Raccanelli, and A. G. Riess, Phys. Rev. Lett. 116, 201301 (2016).

[6] H. Niikura et al., Nat. Astron. 3, 524 (2019).

[7] Y. Ali-Haïmoud and M. Kamionkowski, Phys. Rev. D 95, 043534 (2017).

[8] K. Jedamzik, Phys. Rev. D 55, R5871 (1997).

[9] K. Jedamzik, Phys. Rep. 307, 155 (1998).

[10] T. Bhattacharya et al., Phys. Rev. Lett. 113, 082001 (2014).

[11] C. Schmid, D. J. Schwarz, and P. Widerin, Phys. Rev. Lett. 78, 791 (1997).

[12] C. T. Byrnes, M. Hindmarsh, S. Young, and M. R. S. Hawkins, J. Cosmol. Astropart. Phys. 08 (2018) 041.

[13] R. D. Pisarski and F. Wilczek, Phys. Rev. D 29, 338 (1984).

[14] M. D’Elia, Nucl. Phys. A982, 99 (2019).

[15] B. Holdom, New J. Phys. 10, 053040 (2008).

[16] S. Narison, Phys. Lett. B 693, 559 (2010); 705, 544(E) (2011).

[17] T. Boeckel and J. Schaffner-Bielich, Phys. Rev. Lett. 105, 041301 (2010); 106, 069901 (2011); Phys. Rev. D 85, 103506 (2012).

[18] D. J. Schwarz and M. Stuke, J. Cosmol. Astropart. Phys. 11 (2009) 025; 10 (2010) E01.

[19] S. Ipek and T. M. P. Tait, Phys. Rev. Lett. 122, 112001 (2019).

[20] G. Servant, Phys. Rev. Lett. 113, 171803 (2014).

[21] S. Iso, P. D. Serpico, and K. Shimada, Phys. Rev. Lett. 119, 141301 (2017).

[22] S. Arunasalam, A. Kobakhidze, C. Lagger, S. Liang, and A. Zhou, Phys. Lett. B 776, 48 (2018).

[23] J. H. Applegate and C. J. Hogan, Phys. Rev. D 31, 3037 (1985); 34, 1938(E) (1986).

[24] I. S. Suh and G. J. Mathews, Phys. Rev. D 58, 025001 (1998).

[25] I. S. Suh and G. J. Mathews, Phys. Rev. D 58, 123002 (1998).
[26] D. Boyanovsky, H. J. de Vega, and D. J. Schwarz, Annu. Rev. Nucl. Part. Sci. 56, 441 (2006).

[27] M. B. Christiansen and J. Madsen, Phys. Rev. D 53, 5446 (1996).

[28] B. J. Carr, K. Kohri, Y. Sendouda, and J. Yokoyama, Phys. Rev. D 81, 104019 (2010).

[29] T. Nakama, J. Silk, and M. Kamionkowski, Phys. Rev. D 95, 043511 (2017).

[30] M. Tanabashi et al. (Particle Data Group), Phys. Rev. D 98, 030001 (2018).

[31] J. Braun and H. Gies, J. High Energy Phys. 06 (2006) 024.

[32] M. Sasaki, T. Suyama, T. Tanaka, and S. Yokoyama, Phys. Rev. Lett. 117, 061101 (2016); 121, 059901(E) (2018).

[33] M. Raidal, V. Vaskonen, and H. Veermäe, J. Cosmol. Astropart. Phys. 09 (2017) 037.

[34] Y. Ali-Haïmoud, E. D. Kovetz, and M. Kamionkowski, Phys. Rev. D 96, 123523 (2017).

[35] M. Raidal, C. Spethmann, V. Vaskonen, and H. Veermäe, J. Cosmol. Astropart. Phys. 02 (2019) 018.

[36] S. Knapen, T. Lin, and K. M. Zurek, Phys. Rev. D 96, 115021 (2017).

[37] J. Bergé, P. Brax, G. Métris, M. Pernot-Borràs, P. Touboul, and J. P. Uzan, Phys. Rev. Lett. 120, 141101 (2018).

[38] S. S. Gubser and P. J. E. Peebles, Phys. Rev. D 70, 123511 (2004).

[39] M. A. Shifman, A. I. Vainshtein, and V. I. Zakharov, Nucl. Phys. B147, 448 (1979).

[40] M. A. Shifman, A. I. Vainshtein, and V. I. Zakharov, Nucl. Phys. B147, 385 (1979).

[41] P. Gubler and D. Satow, Prog. Part. Nucl. Phys. 106, 1 (2019).

[42] E. Witten, Phys. Rev. D 30, 272 (1984).

[43] C. Caprini, R. Durrer, and X. Siemens, Phys. Rev. D 82, 063511 (2010).

[44] P. Schwaller, Phys. Rev. Lett. 115, 181101 (2015). 